\title{
X-ray coherent diffraction imaging with an objective lens: Towards three-dimensional mapping of thick polycrystals
}

\author{
A. F. Pedersen $\odot,{ }^{1,2}$ V. Chamard $\odot,{ }^{3}$ C. Detlefs $\odot,{ }^{4}$ T. Zhou $\odot,{ }^{4}$ D. Carbone, ${ }^{5}$ and H. F. Poulsen $\odot{ }^{1, *}$ \\ ${ }^{1}$ Department of Physics, Technical University of Denmark, 2800 Kongens Lyngby, Denmark \\ ${ }^{2}$ Xnovo Technology ApS, 4600 Koge, Denmark \\ ${ }^{3}$ Aix Marseille Univ, CNRS, Centrale Marseille, Institut Fresnel, 13013 Marseille, France \\ ${ }^{4}$ European Synchrotron Radiation Facility, 71 Avenue des Martyrs, 38000 Grenoble, France \\ ${ }^{5}$ MAX IV Laboratory, Fotongatan 2, 22592 Lund, Sweden
}

(Received 3 March 2019; revised 8 January 2020; accepted 21 April 2020; published 7 July 2020)

\begin{abstract}
We demonstrate an x-ray coherent imaging method that combines high spatial resolution with the ability to map grains within thick polycrystalline specimens. An x-ray objective serves to isolate an embedded grain. Iterative oversampling routines and Fourier synthesis are used to reconstruct the shape and strain field from the far-field intensity pattern. In a demonstration experiment a $\sim 500-\mathrm{nm}$ Pt grain embedded in a polycrystalline $\mathrm{Pt}$ matrix is mapped in three dimensions without compromising the spatial resolution. No information on the pupil function of the lens is required and lens aberrations are not critical.
\end{abstract}

DOI: 10.1103/PhysRevResearch.2.033031

\section{INTRODUCTION}

In polycrystalline materials, the three-dimensional (3D) distribution of strain, defects, and lattice distortions within the individual grains strongly affects the macroscopic mechanical and physical properties. To understand this relation, 3D mapping of the local structure and strain and its evolution during processing is required-and the sample volume studied should be representative of the hierarchically organized structure. Current imaging methods all involve compromises. Electron microscopy methods such as transmission electron microscopy TEM [1] and Electron BackScatter Diffraction (EBSD) [2] are destructive techniques and cannot probe the strain in general. Nondestructive 3D grain mapping of millimeter-sized samples can be carried out by x-ray diffraction tomography methods [3-6] with a resolution of $2 \mu \mathrm{m}$. These may be complemented by dark-field $\mathrm{x}$-ray microscopy [7-9] where an x-ray objective lens is placed in the Bragg diffracted beam of a selected grain. This magnifies the projection image and acts as a very efficient filter, removing overlap of diffraction from other grains. Aberrations of refractive $\mathrm{x}$ ray lenses, however, currently limit the resolution of dark-field x-ray microscopy to approximately $100 \mathrm{~nm}$.

$\mathrm{X}$-ray coherent diffraction imaging (CDI) has emerged as a powerful tool for 3D mapping of small isolated samples [10-14]. The method retrieves the sample scattering function from a set of coherent intensity measurements, using

\footnotetext{
*hfpo@fysik.dtu.dk
}

Published by the American Physical Society under the terms of the Creative Commons Attribution 4.0 International license. Further distribution of this work must maintain attribution to the author(s) and the published article's title, journal citation, and DOI. computational inversion approaches to determine the phase of the scattered amplitude, which cannot be directly measured by the detector. The sample image is obtained by backpropagation of the scattered field. For crystalline specimens, the intensity distribution can be measured in the vicinity of a chosen Bragg peak [12-16], resulting in maps of the material density and of the atomic displacement projected along the Bragg vector. Existing (Bragg) CDI methods do not employ any optical elements between the sample and the detector. Compared to full-field microscopy methods using an objective lens between the sample and the detector, CDI thus avoids limitations due to aberrations and finite aperture of the lens, and spatial resolutions in the $10-\mathrm{nm}$ range can be obtained with strain sensitivity on the order of a few times $10^{-4}$ [17]. The use of Bragg CDI (BCDI) has been, until now, limited to (sub-) micrometer-sized isolated crystals exhibiting rather limited strain fields. Bragg ptychography pushes the method to laterally extended crystals and larger strain fields $[17,18]$. Nevertheless, these approaches are still restricted to samples with a relatively limited complexity, and very often an ad hoc sample preparation is mandatory for the success of the experiment. For studies of thick polycrystalline samples, none of these approaches is applicable, because such samples typically contain a large number of simultaneously diffracting micro- or nanocrystals within the illuminated volume. Their individual diffraction patterns are incoherently superposed, which makes the phase reconstruction impossible.

Here, we experimentally demonstrate a method that is capable of combining the spatial resolution of BCDI with the ability of dark-field x-ray microscopy to study a selected, embedded grain or domain within a thick polycrystalline sample. The method, referred to as objective-based BCDI (or objective BCDI), can be combined with the coarser grain mapping methods described above, enabling multiscale 


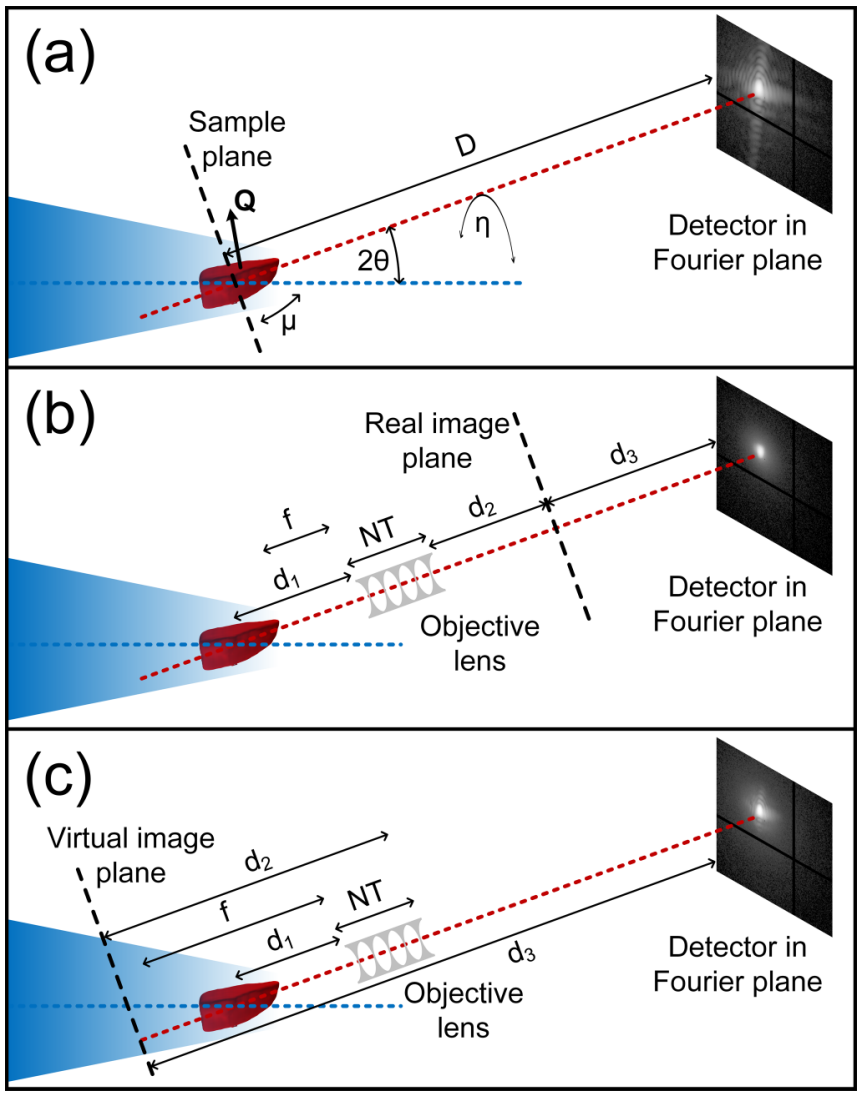

FIG. 1. Optical setup principles. (a) Classical BCDI: The detector monitors the far-field diffraction produced by the sample, with no intervening optical elements. The optical axis of the diffracted beam is characterized by angles $(2 \theta, \eta)$. (b) Objective-based BCDI with a real image plane (working distance $d_{1}$ greater than focal length $f$ ). The objective is a compound refractive lens with $N$ lenslets, each of thickness $T$. (c) Objective-based BCDI with a virtual image plane $\left(d_{1}<f\right)$. In all cases, the 3D reconstructions are based on images acquired at a set of tilt angles, $\mu$.

microscopy in 3D for crystalline materials. As illustrated in Fig. 1, we can use a dark-field microscope to create either a real or a virtual image of the diffracting object. The diffraction pattern is then recorded in the far-field Fraunhofer limit. The probing volume is restricted either by the field of view of the objective itself - which can be varied by the design of the objective-or by inserting an aperture in the image plane. As shown numerically in Ref. [19] on a phantom, the introduction of a set of lenses does not modify the intensity distribution in the far field of the sample (except for scaling), as long as the related aberrations manifest themselves as an additional phase field and that the lenses are placed at a large enough distance from the sample, i.e., in the far-field regime of the sample diffracted field. Thereby, the intensity in the detector plane is fully consistent with the one obtained without a lens. This allows the use of existing iterative BCDI algorithms to retrieve the sample crystalline properties [19]. In this paper we demonstrate these optical properties experimentally. Furthermore, we show that the spatial resolution limitation introduced by the finite numerical aperture of the objective can be overcome by the introduction of Fourier synthesis approaches [20], applied in the X-ray regime.

\section{EXPERIMENTAL IMPLEMENTATION}

The experiment was carried out at beamline ID01 of the European Synchrotron Radiation Facility (ESRF) using a compound refractive lens (CRL) [21] as the objective. Selecting a $~ 500$-nm-sized Pt grain within a 4- $\mu$ m-thin Pt foil, rather than one buried in a bulk polycrystal, allowed us to make a direct comparison of our objective BCDI method with classical BCDI by translating the objective in and out of the diffracted beam, respectively. The aim of this experiment was to demonstrate the feasibility of the approach and to detect and characterize any deviations arising from introducing the objective. In all configurations presented in Fig. 1, the full 3D intensity pattern is derived from a set of two-dimensional (2D) patterns acquired during a "rocking scan," where the sample is tilted at equidistant angular positions around the axis $\mu$, perpendicular to the incoming beam and to the Bragg vector (see Fig. 1).

Key optical parameters and details of the geometry definitions are presented in Appendix A. The implemented microscope was aligned with the optical axis corresponding to the $\operatorname{Pt}(111)$ Bragg diffracted beam, at $2 \theta \sim 40^{\circ}$, for an 8 -keV coherent beam. The CRL comprised $N$ identical Be biparaboloid lenslets with an apex radius of curvature of $50 \mu \mathrm{m}$ placed a distance $T=2 \mathrm{~mm}$ apart. $N$ was 70 and 20 in the real and virtual image configuration, respectively. Using geometrical optics expressions for a thick lens [8] the distances were configured to match a magnification of 1.4 and -0.9 , respectively. The actual magnification was determined with high precision by comparing the fringe pattern to the BCDI case. Notably, the Fresnel number $N_{F}$ is small (see last column in Table II) for the two objective BCDI configurations, demonstrating that the objective lens is placed in the far-field limit.

\section{RESULTS WITHOUT FOURIER SYNTHESIS}

Corresponding raw data for the three configurations are shown in Fig. 2. As further illustrated in Appendix B, they are similar, with the exception of the anticipated cutoffs caused by the different numerical apertures. Reconstructions were in all cases performed by using a combination of the hybrid input-output algorithm (HIO) [22] with a feedback parameter of $\beta=0.9$, the error reduction algorithm (ER) [23], and the shrinkwrap algorithm [24] to update the support. The resulting maps-shown in Fig. 2-demonstrate good agreement between the no-objective case and the objective-based approach. The corresponding spatial resolutions (for details, see Appendix C) are tabulated in Table I. In Appendixes D and $\mathrm{E}$ we show that most of the differences can be explained by the bandpass defined by the aperture, with the remaining discrepancies being smaller than the estimated error bars. This is further corroborated by the fact that there is no noticeable difference along the third direction in reciprocal space, i.e., the rocking direction, where there is no cutoff and for which the spatial resolution is very similar to the one obtained for the no-lens case. This experimentally confirms our recent numerical results [19]. 


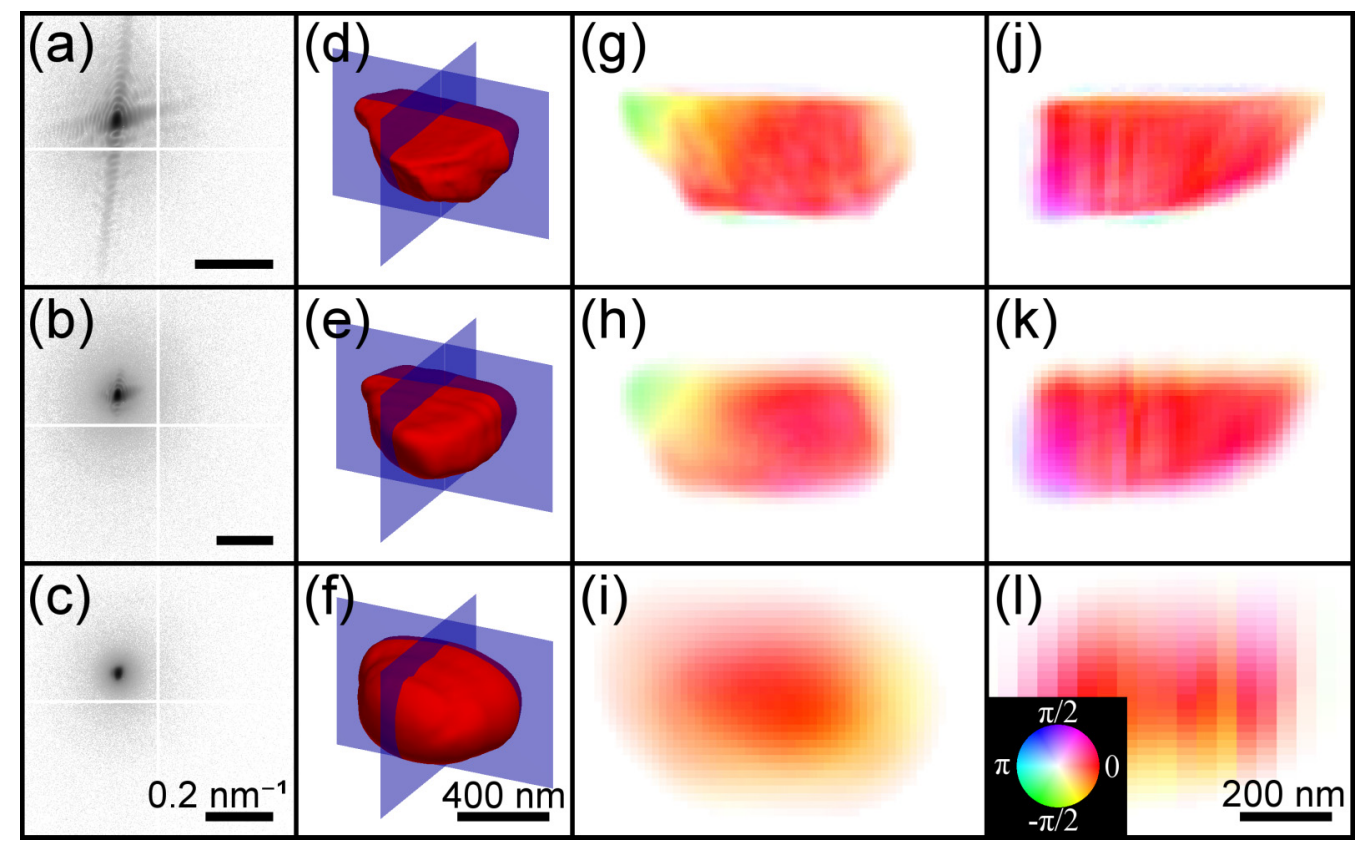

FIG. 2. Reconstruction of a single Pt grain obtained without Fourier synthesis. Raw data taken at the maximum of the rocking curve (first column), resulting 3D shapes of the selected grain (second column), and phase reconstructions for the two blue planes indicated (third and fourth columns). The rows represent classical BCDI (top), objective BCDI with a virtual image plane (middle), and objective BCDI with a real image plane (bottom). The scale bars in (a)-(c) have the same Fourier space length indicated in (c), a common scale bar for (d)-(f) is shown in (f), and a common scale bar for (g)-(l) in (1). The images (g)-(l) display the reconstructed complex fields, with the phase represented by the color and the amplitude indicated by the intensity (hue); see the color wheel inset in (1).

\section{RESULTS WITH FOURIER SYNTHESIS}

To increase the effective bandpass for the virtual image configuration [Fig. 1(c)], we repeated the data acquisition over a $3 \times 3$ grid along the $2 \theta$ and $\eta$ directions (see Appendix F). The grid step size of $0.1^{\circ}$ is 0.3 times the numerical aperture of the objective lens, corresponding to a $40 \%-70 \%$ overlap of regions (Appendix G).

Two Fourier synthesis algorithms are introduced for the inversion of the nine $3 \mathrm{D}$ data sets. Flow charts are provided in Appendix $\mathrm{H}$ and the robustness of both approaches has been numerically confirmed, as shown in Appendix I. In the first one, the data sets are treated in parallel, stitching the complex field in Fourier space by taking the mean of the aligned reconstructions. This reconstruction method is robust, as it does not rely on any a priori information about the pupil size or the exact position of the objective. The results, shown in Fig. 3, exhibit a spatial resolutiontabulated in Table I-which, within experimental error, is as good as or slightly better than the classical BCDI solution. More importantly, as seen by comparing Figs. 3(j) and 3(k) with Figs. 2(g) and 2(j), the resulting phase reconstruction is remarkably similar to that of classical BCDI. (One cannot exclude, however, that there are small discrepancies due to slight variations of the incoming beam wavefront, during the beam time.) Additional comparisons in Fourier space are provided in Appendix $\mathrm{J}$.

The second algorithm is based on updating sections of the global reconstructed Fourier plane with the measurements in a serial fashion. This method requires knowledge of the pupil function. The results are shown in Figs. 3(1) and $3(\mathrm{~m})$, for direct comparison with Figs. 3(j), 3(k), 2(g), and $2(\mathrm{j})$. The spatial resolution is tabulated in Table I. It is comparable to the no-lens case but slightly degraded with respect to the parallel synthesis case. Artifacts also appear, e.g., on the left edge in Fig. 3(1). We hypothesize that this is due to shot noise induced inconsistencies between the nine data sets. As the object is updated in a sequential manner, those inconsistencies are not averaged, contrary to the serial case. They likely produce stagnation in the inversion process, which is not stable enough to allow support updating using the shrinkwrap algorithm, as we observed. Hence, for this experiment, we consider the parallel synthesis to provide a better reconstruction.

TABLE I. Estimation of the spatial resolution in 3D. See Appendix C for details of the calculation.

\begin{tabular}{lccccc}
\hline \hline Parameter & No lens & Real image & Virtual image & Parallel synthesis & Serial synthesis \\
\hline Resolution, $x$ & $39 \mathrm{~nm}$ & $128 \mathrm{~nm}$ & $51 \mathrm{~nm}$ & $27 \mathrm{~nm}$ & $37 \mathrm{~nm}$ \\
Resolution, $y$ & $30 \mathrm{~nm}$ & $131 \mathrm{~nm}$ & $54 \mathrm{~nm}$ & $35 \mathrm{~nm}$ & $34 \mathrm{~nm}$ \\
Resolution, $z$ & $43 \mathrm{~nm}$ & $170 \mathrm{~nm}$ & $48 \mathrm{~nm}$ & $32 \mathrm{~nm}$ & $45 \mathrm{~nm}$ \\
\hline \hline
\end{tabular}




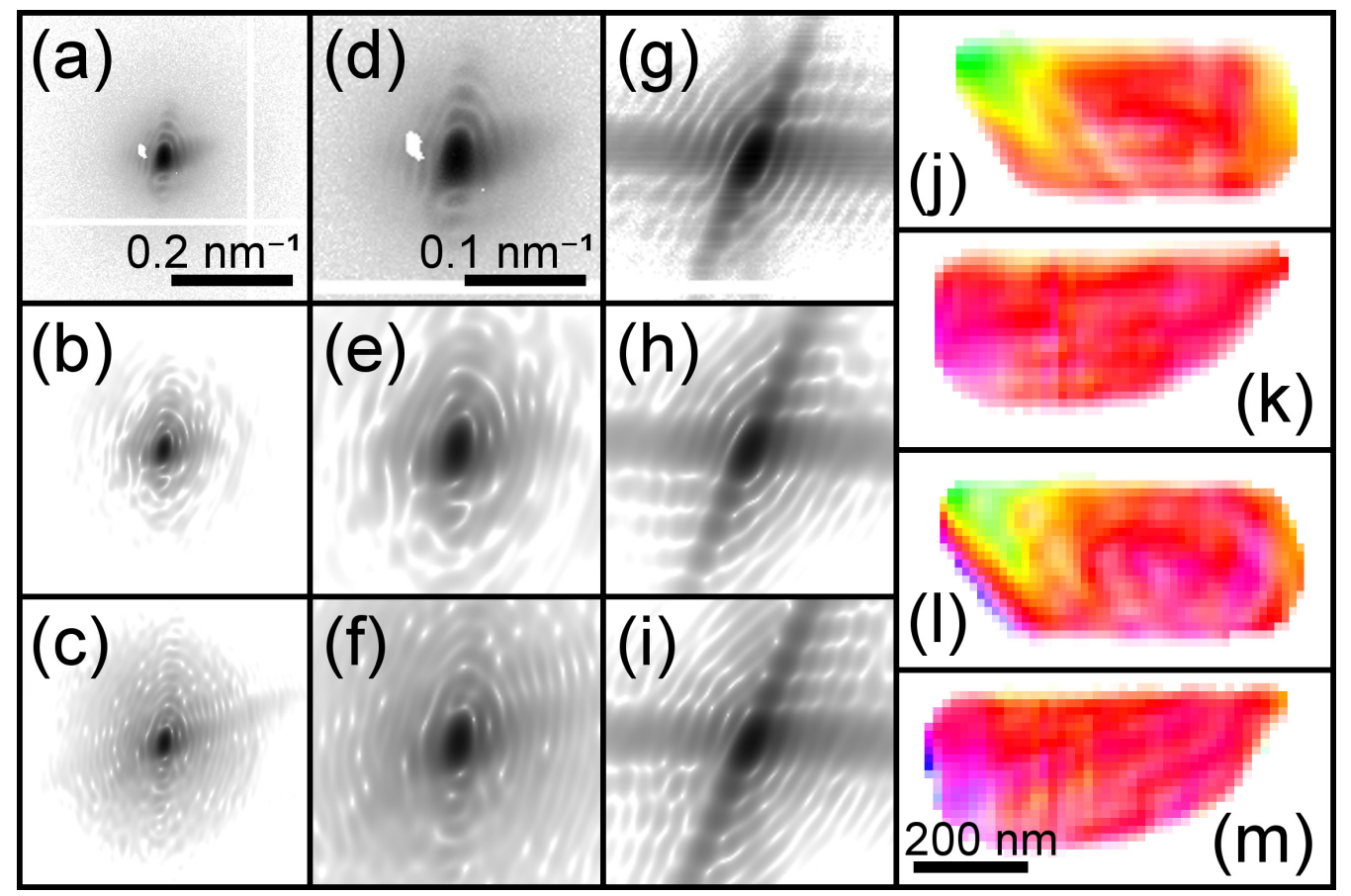

FIG. 3. Reconstructions obtained using synthesis in Fourier space for objective BCDI with a virtual image plane. (a)-(c) Intensity distribution at the center of the rocking curve $\left(\mu=0^{\circ}\right)$ in the $\left(q_{1}, q_{2}\right)$ detector plane: (a) raw data from a single data set; (b) the reconstructed Fourier intensity using parallel synthesis; (c) the reconstructed Fourier intensity using serial synthesis. (d) $-(\mathrm{f})$ are zoomed $(\times 2)$ images of (a)-(c). (g)-(i) are zoomed Fourier space $\left(q_{1}-q_{3}\right.$ plane), at the same $q$ scale as (d)-(f). In (a)-(i), the scale is logarithmic and covers six orders of magnitude (one photon to 1000000 photons). (j), (k) Reconstructions of two sections in the grain using parallel synthesis; (1), (m) reconstructions using serial synthesis. For direct comparison with results in Fig. 2, (a)-(c) have the same scale, as do (j)-(m). Additional comparisons in Fourier space are given in Appendix J.

For both Fourier synthesis methods, we observe that a large overlap in Fourier space is not necessary for a successful reconstruction (see Appendix G).

The approach presented herein shares some roots with, e.g., Fourier ptychography [25-27] and phase contrast confocal microscopy [28]. Contrary to Fourier ptychography, however, where the overlapping scanning space and the measurement spaces are conjugated spaces, and which requires a large amount of overlap, our Fourier synthesis method uses the overlapping scans performed in the measurement space and works for a limited amount of overlap. Interestingly, the configuration with a real image plane enables the recording of preliminary images in both direct space and Fourier space (in the image plane and in the back focal plane or in the far-field diffraction plane, respectively). This allows combined reconstructions and the use of a priori knowledge about the sample, such as support constraints. We believe such flexibility could be a route towards higher resolution in both direct and Fourier domains, thereby opening the CDI methods towards the high-resolution imaging of samples exhibiting highly complex strain variations.

\section{CONCLUSION}

We have demonstrated a technique that generalizes the capability of CDI approaches for the 3D measurement of shape and strain distribution of isolated structures to the study of grains embedded in a polycrystalline material. The possibility of measuring deeply embedded grains is limited solely by the penetration depth of the radiation used. The method is intended to be used with next-generation synchrotrons, providing high-coherence beams at higher $\mathrm{x}$-ray energies. With hard x-rays even a thick sample can be considered weakly scattering, and we expect the phase shift induced by the strain

TABLE II. Key optical parameters. $N$ is the number of lenslets. Distances $d_{1}, d_{2}, d_{3}$ are defined in Fig. $1 . M$ is the magnification. The numerical aperture (NA) represents the FWHM of the angular acceptance of the detector (for BCDI) or the CRL (for objective BCDI). In order to compare with a no-lens BCDI setup, the effective sample-detector distance $D_{\text {eff }}$ is given $\left(D_{\text {eff }}=d_{3} / M\right)$, corresponding to the distance at which the intensity patterns on a hypothetical detector have the same scale as the BCDI configuration. $\sigma_{\text {pupil }}$ is the rms width of the Gaussian pupil function. $N_{F}$ is the Fresnel number at the entrance of the lens, assuming a $500 \mathrm{~nm}$ aperture (sample).

\begin{tabular}{|c|c|c|c|c|c|c|c|c|c|}
\hline & & $d_{1}(\mathrm{~m})$ & $d_{2}(\mathrm{~m})$ & $d_{3}(\mathrm{~m})$ & $M$ & NA (mrad) & $D_{\text {eff }}(\mathrm{m})$ & $\sigma_{\text {pupil }}($ pixel $)$ & $N_{F}$ \\
\hline Bragg CDI & - & - & - & - & - & $\sim 13.5$ & 1.632 & - & - \\
\hline Objective BCDI, real image & 0 & 0.121 & 0.100 & 1.271 & -0.899 & 1.44 & 1.414 & 7.85 & 0.013 \\
\hline Objective BCDI, virtual image & 20 & 0.050 & -0.119 & 1.661 & 1.409 & 5.5 & 1.179 & 25.2 & 0.032 \\
\hline
\end{tabular}


distribution and lattice rotation to act predominantly on the diffracted beam. Compared to state-of-the-art dark-field x-ray microscopy, the spatial resolution can be improved by a factor of at least 5 and is only limited by data acquisition time and experimental stability. This work is a proof of concept; the optimization of experimental geometry and reconstruction algorithms as well as applications on thick specimens will be detailed in the future, as soon as a high-energy highly intense highly coherent $x$-ray beam becomes available at a BCDI beamline. In particular, the recent manufacture of high-quality multilayer Laue lenses [29] makes it possible to employ hard $\mathrm{X}$-ray objectives with a higher numerical aperture [30]. Moreover, the setup presented is easily combined with both darkfield $\mathrm{x}$-ray microscopy and coarse grain mapping of the entire specimen. This approach should enable the investigation of crystalline domains in hierarchically organized structures such as most metals, ceramics, rocks, ice, sand, bones, and many artifacts of artistic and archaeological interest.

\section{ACKNOWLEDGMENTS}

We acknowledge funding support from ERC Advanced Grant No. 291321 and ERC Consolidator Grant No. 724881. We also acknowledge funding from DanMAX, Grant No. 4059-00009B. We thank Y. Zhang and J. Bowen for help with providing the sample, H. Simons, M. Beltran, and J. W. Andreasen for scientific discussions, ESRF for provision of beam time (MI-1315), and the instrument center Danscatt for a travel grant.

\section{APPENDIX A: EXPERIMENTAL GEOMETRY AND FRAME DEFINITIONS}

The generics of the experimental geometry are shown in Fig. 4. The objective itself has an extension of $N T$, where $N$ is the number of lenses and $T$ the distance between lens centers. Note that the distance $d_{2}$ is the distance between the lens and the real or virtual image plane and $d_{3}$ is the distance between

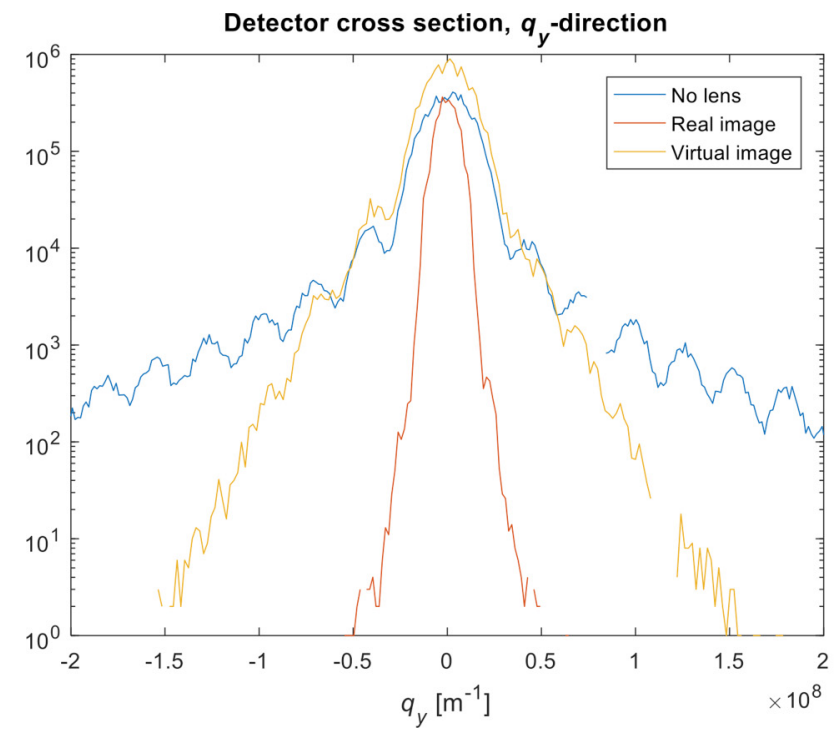

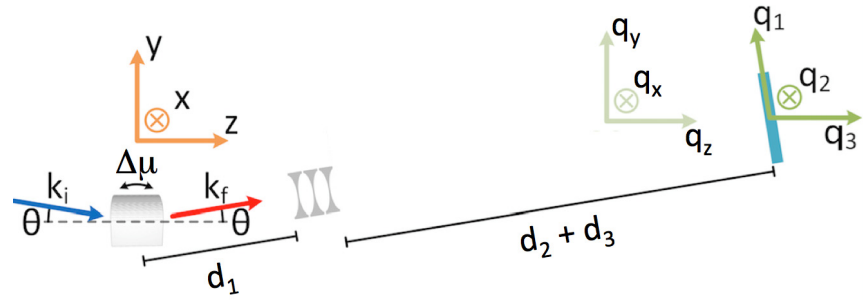

FIG. 4. BCDI experimental geometry and definition of the measurement and reconstruction frames.

the image plane and the detector. For the virtual image plane geometry, $d_{2}$ becomes negative.

The different frames (coordinate systems) used in the study either for measurement or as part of the reconstruction procedure are also illustrated: $\left(q_{1}, q_{2}, q_{3}\right)$ is the nonorthogonal measurement frame; $\left(q_{x}, q_{y}, q_{z}\right)$ is an orthogonal frame in reciprocal space, chosen so that $q_{x}=q_{2}$ and $q_{z}=q_{3} ;(x$, $y, z)$ is the frame conjugated to $\left(q_{x}, q_{y}, q_{z}\right)$, according to Fourier conjugation relations. $\theta$ is the scattering angle, and $\Delta \mu$ represents the offset from the maximum position of the rocking curve.

For the experiment, a combination of a Si(111) BraggBragg monochromator, a set of slits, and a Kirkpatrick-Baez (KB) mirror used as a condenser generated a fully coherent $8-\mathrm{keV}$ incoming beam with dimensions of $1.5 \times 2.0 \mu \mathrm{m}^{2}$ (Full width at half maximum) at the sample position. The acquisition was performed with a far-field Maxipix detector with $516 \times 516$ pixels of $55 \mu \mathrm{m}$ size. A series of $2 \mathrm{D}$ patterns was measured along the rocking curve, with rocking step size $\Delta \mu=0.003^{\circ}$. Key optical parameters are presented in Table II.

\section{APPENDIX B: COMPARISON OF THE INTENSITY DISTRIBUTIONS FOR BCDI AND OBJECTIVE BCDI}

Complementary to Fig. 2, Fig. 5 provides a better visualization of the impact of the lens on the measured intensity

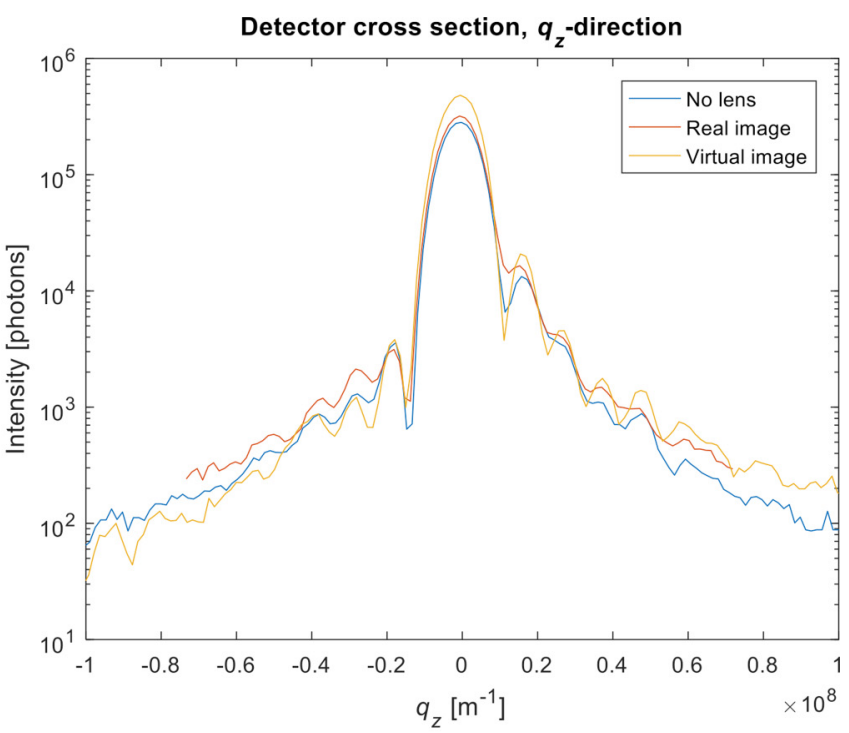

FIG. 5. Intensity distributions for BCDI and objective BCDI. 1D cross sections are compared, along two main directions of reciprocal space, for the no-lens BCDI, the real image objective BCDI, and the virtual image BCDI experiments. 
TABLE III. Estimation of the spatial resolution in 3D, using the PRTF approach. The spatial resolutions along the main three space directions are given, resulting from the Gaussian fit of the PRTF function, for the classical no-lens BCDI experiment, and the two objective-based BCDI experiments (real image and virtual image). In addition, the voxel sizes in real- and reciprocal space are detailed. The axis directions refer to the definitions given in Appendix A.

\begin{tabular}{lccc}
\hline \hline Parameter & No lens & Real image & Virtual image \\
\hline Resolution (PRTF), $x$ & $38 \mathrm{~nm}$ & $61 \mathrm{~nm}$ & $25 \mathrm{~nm}$ \\
Resolution (PRTF), $y$ & $16 \mathrm{~nm}$ & $69 \mathrm{~nm}$ & $28 \mathrm{~nm}$ \\
Resolution (PRTF), $z$ & $28 \mathrm{~nm}$ & $90 \mathrm{~nm}$ & $28 \mathrm{~nm}$ \\
Voxel size real-space, $x$ & $9.0 \mathrm{~nm}$ & $15.6 \mathrm{~nm}$ & $13.0 \mathrm{~nm}$ \\
Voxel size real-space, $y$ & $9.6 \mathrm{~nm}$ & $16.6 \mathrm{~nm}$ & $13.8 \mathrm{~nm}$ \\
Voxel size real-space, $z$ & $15.5 \mathrm{~nm}$ & $43.3 \mathrm{~nm}$ & $15.5 \mathrm{~nm}$ \\
Voxel size reciprocal space, $x$ & $1.37 \mu \mathrm{m}^{-1}$ & $1.58 \mu \mathrm{m}^{-1}$ & $1.89 \mu \mathrm{m}^{-1}$ \\
Voxel size reciprocal space, $y$ & $1.37 \mu \mathrm{m}^{-1}$ & $1.58 \mu \mathrm{m}^{-1}$ & $1.89 \mu \mathrm{m}^{-1}$ \\
Voxel size reciprocal space, $z$ & $1.45 \mu \mathrm{m}^{-1}$ & $1.45 \mu \mathrm{m}^{-1}$ & $1.45 \mu \mathrm{m}^{-1}$ \\
Number of voxels, $x$ & 512 & 256 & 256 \\
Number of voxels, $y$ & 512 & 256 & 256 \\
Number of voxels, rocking & 280 & 100 & 280 \\
\hline \hline
\end{tabular}

distribution. To this aim, one-dimensional (1D) cross sections are presented along two main directions of the reciprocal space. In the detector plane ( $q_{y}$, left panel), the lenses manifest themselves as a decrease of intensity at large $q_{y}$ values. For the real image case, the effect is so strong that the interference fringes are not visible anymore. For the virtual image case, the fringes are attenuated, but their width and positions are unaffected, confirming the absence of aberration effects. Interestingly, along the rocking curve direction (i.e., the $q_{z}$ direction, right panel), the three intensity distributions are comparable, as expected.

\section{APPENDIX C: ESTIMATION OF THE SPATIAL RESOLUTION IN 3D}

The spatial resolution is first estimated with the help of the phase retrieval transfer function (PRTF) [31]. It requires the comparison of different reconstructions obtained as follows: The initial guess was generated in Fourier space by taking the measured amplitude and combining it with a random phase. For each BCDI experiment (no-lens BCDI, real image objective BCDI, and virtual image objective BCDI) a total of 20 reconstructions were produced, from which the ten best ones (lowest reconstruction error) were selected and used to calculate the PRFT (after aligning the objects in real-space and taking the overall phase factor into account in Fourier space). Finally, a Gaussian fit was applied to the PRTF along the $x, y$, and $z$ directions. The found peak width was used to determine the 1/e threshold, as usual, arbitrarily chosen [31]. The results are reported in Table III, together with the other key voxel size parameters.

However, the PRTF analysis is a measurement of the data fidelity: It describes how well the reconstructions fit the data. This can be misleading for truncated data, such as the ones
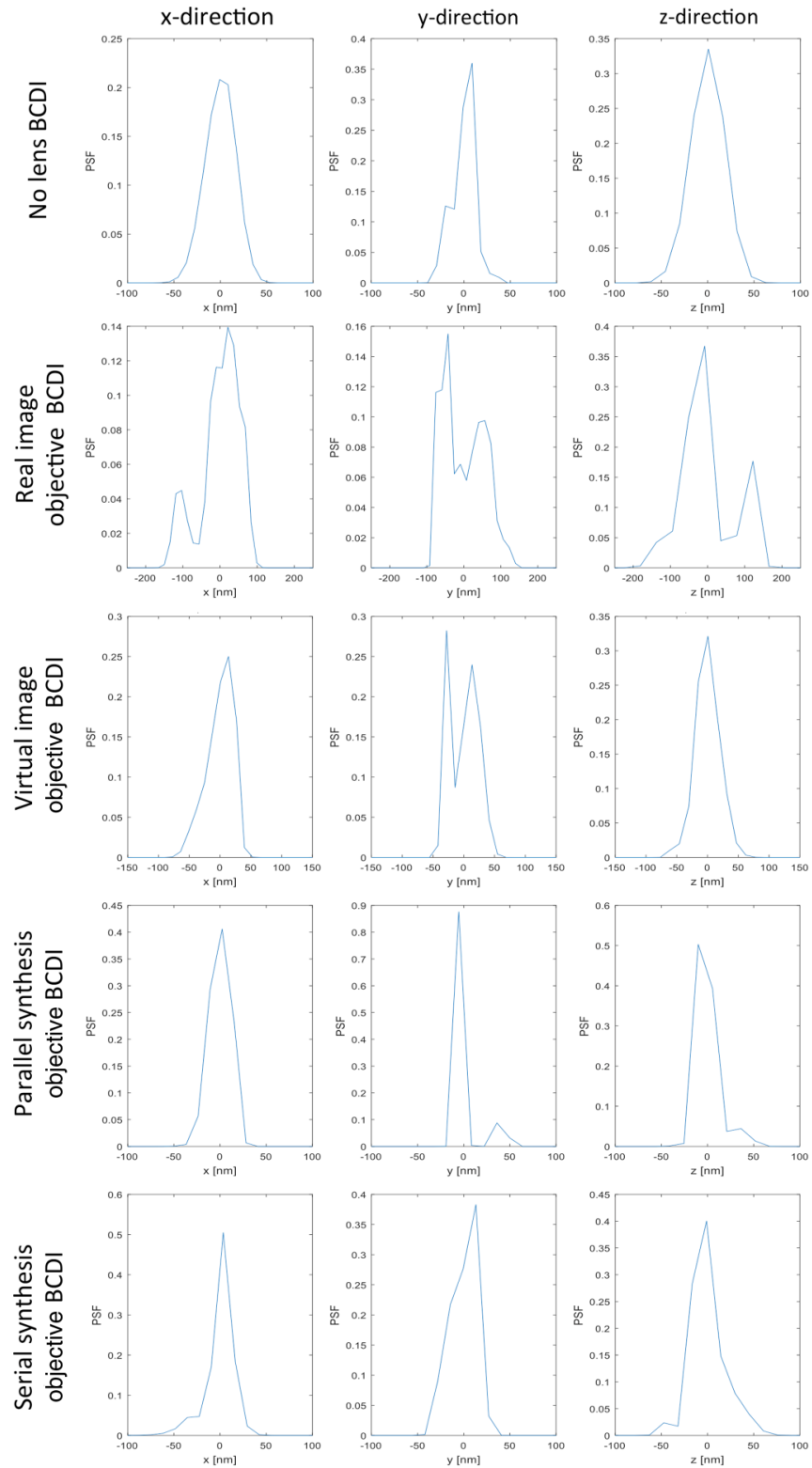

FIG. 6. Results of the deconvolution approach used for estimating the spatial resolution in 3D. The blurring function as described in Ref. [32] is plotted along the three main space directions, for all experimental approach reconstructions.

obtained with the finite-size lens aperture. It translates here into a surprisingly "good" resolution power for the virtual image case (Table III). To circumvent this problem, we apply the approach proposed by Cherukara et al. [32]. This new method, based on a deconvolution process, works in realspace and is therefore well suited to take the aperture into account. Consequently, it can also be applied to the Fourier synthesis approaches. Results of the deconvolution procedure are shown in Fig. 6 while the resulting estimates of the spatial resolution values along the three main directions are given in Table I. The good agreement between this approach and the PRTF approach for the no-lens case validates the consistency between the two resolution estimation tools. 
As expected for the real and virtual image experiments, the spatial resolution is degraded in the $(x, y)$ plane. Along the $z$ direction, the resolution is almost unaffected by the lens, as this direction corresponds to the direction of the rocking curve. For the real image case, only 100 rocking steps were used instead of 280, explaining the degradation of the resolution also along the $z$ direction. For the Fourier synthesis approaches, the spatial resolution is preserved and even slightly improved for the parallel synthesis reconstruction. All reconstructions presented in the main text are based on this approach.

\section{APPENDIX D: SIMULATION OF THE EFFECT OF THE APERTURE}

To simulate the effect of the pupil function on the classical (lensless) Bragg CDI data we Fourier transform the reconstruction for the classical BCDI [shown in Fig. 7(a)], multiply it by the Gaussian pupil function with the width specified in Table II, and then inverse Fourier transform the result back to real-space. The results using the pupil functions for the virtual and real image geometry are displayed in Figs. 7(b) and 7(c), respectively. These results can be compared directly to the reconstructions with the objective in place, shown in Figs. 2(h) and 2(i), and for convenience reproduced here as Figs. 7(d) and $7(\mathrm{e})$. It is evident that, to a large extent, the discrepancy between the classical BCDI result and the objective CDI results can be explained by the finite pupil.

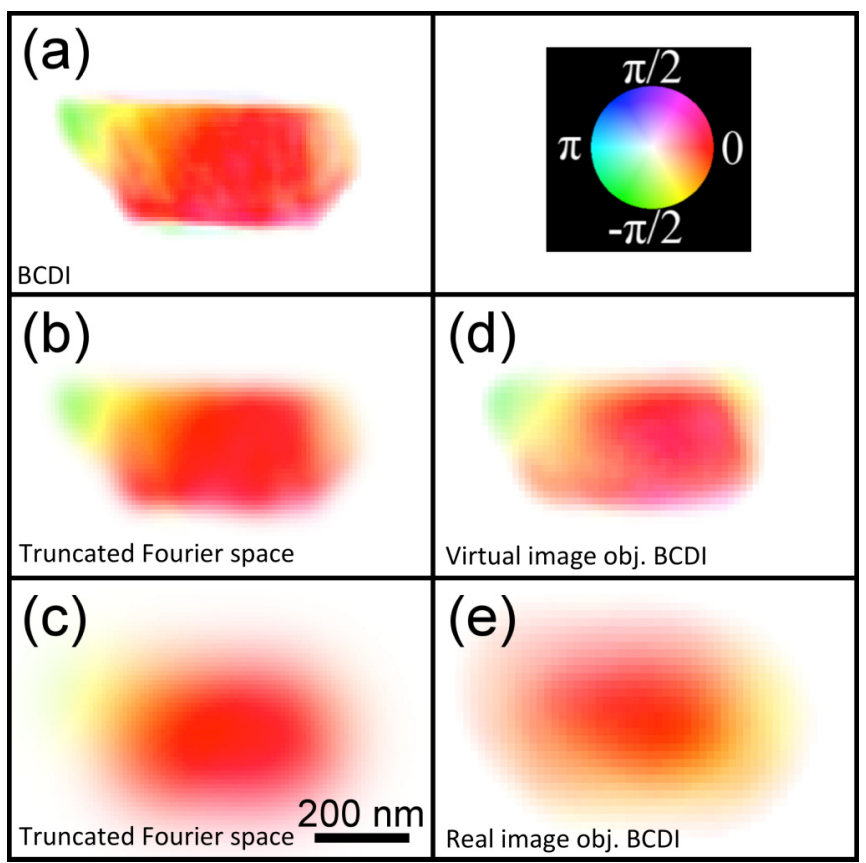

FIG. 7. A study of the effect of the finite pupil on reconstruction quality. The classical BCDI reconstruction in (a) is used as a phantom for numerical studies. Results from applying a pupil of a size corresponding to the virtual image case (b), and the real image case (c) are compared to the reconstructions from the measurements with the lens in the virtual and real image geometry in (d), (e), respectively. All plots share the same scale.

\section{APPENDIX E: ESTIMATION OF THE ERROR BARS OF THE RETRIEVED IMAGES}

In order to provide a standard to compare reconstructions, the variability observed among the reconstructions produced from the same data set but with different starting guesses has been investigated. For each experiment (no-lens BCDI, real image objective $\mathrm{BCDI}$, and virtual image $\mathrm{BCDI}$ ), a set of 20 reconstructions with comparable error metric has been produced. The variability is established as follows:

(1) Align the center of mass of the object amplitudes; i.e., align the objects in real-space.

(2) No lens only: Remove the object phase ramp coming from the Bragg peak not being centered on the detector.

(3) Remove the global phase offset (estimated by averaging the phase in a box of $11 \times 11 \times 11$ voxels).

(4) Normalize the amplitude to the maximum value of the mean amplitude of all 20 reconstructions.

(5) Calculate the mean and standard deviation for the amplitude and phase.

(6) As an overall metric calculate the mean of the standard deviation inside the object. Note that the normalized average amplitude inside the object is greater than 0.3 .

The results are shown in Fig. 8.

\section{APPENDIX F: EXAMPLES OF RAW DATA FOR FOURIER SYNTHESIS}

Shown in Fig. 9 are corresponding examples of raw data for the nine data sets used for the Fourier synthesis in the virtual image geometry. The step size in $2 \theta$ and $\eta$ are both $0.1^{\circ}$. The white lines are gaps between the detector modules.

\section{APPENDIX G: OVERLAPPING RATIO IN THE MEASUREMENT SPACE (FOURIER SYNTHESIS)}

In order to evaluate the impact of the (measurement space) overlapping ratio on the quality of the reconstruction, we have performed two Fourier synthesis reconstructions using only five data sets (instead of nine), corresponding to the four outermost corner data set and the middle one. The corresponding overlapping ratio between two successive $3 \mathrm{D}$ intensity patterns is estimated to about $40 \%-50 \%$ (see Table IV). As observed in Fig. 10, both reconstruction processes (parallel and serial synthesis approaches) result in satisfying reconstructions. As expected, the serial synthesis reconstruction exhibits some visible artifacts. They likely result from inconsistencies in the data sets (induced by photon shot noise), which produce stagnation and instabilities in the reconstruction. Indeed, the serial Fourier synthesis approach involves the retrieval of the object in a sequential manner, in which successive updates arising from the successive data are accounted for.

\section{APPENDIX H: FLOW CHARTS OF THE FOURIER SYNTHESIS ALGORITHMS}

This Appendix presents the details of the two Fourier synthesis algorithms. Corresponding flow charts are provided in Fig. 11.

In the parallel synthesis approach, the data sets are treated in parallel, stitching the complex field in Fourier space by 


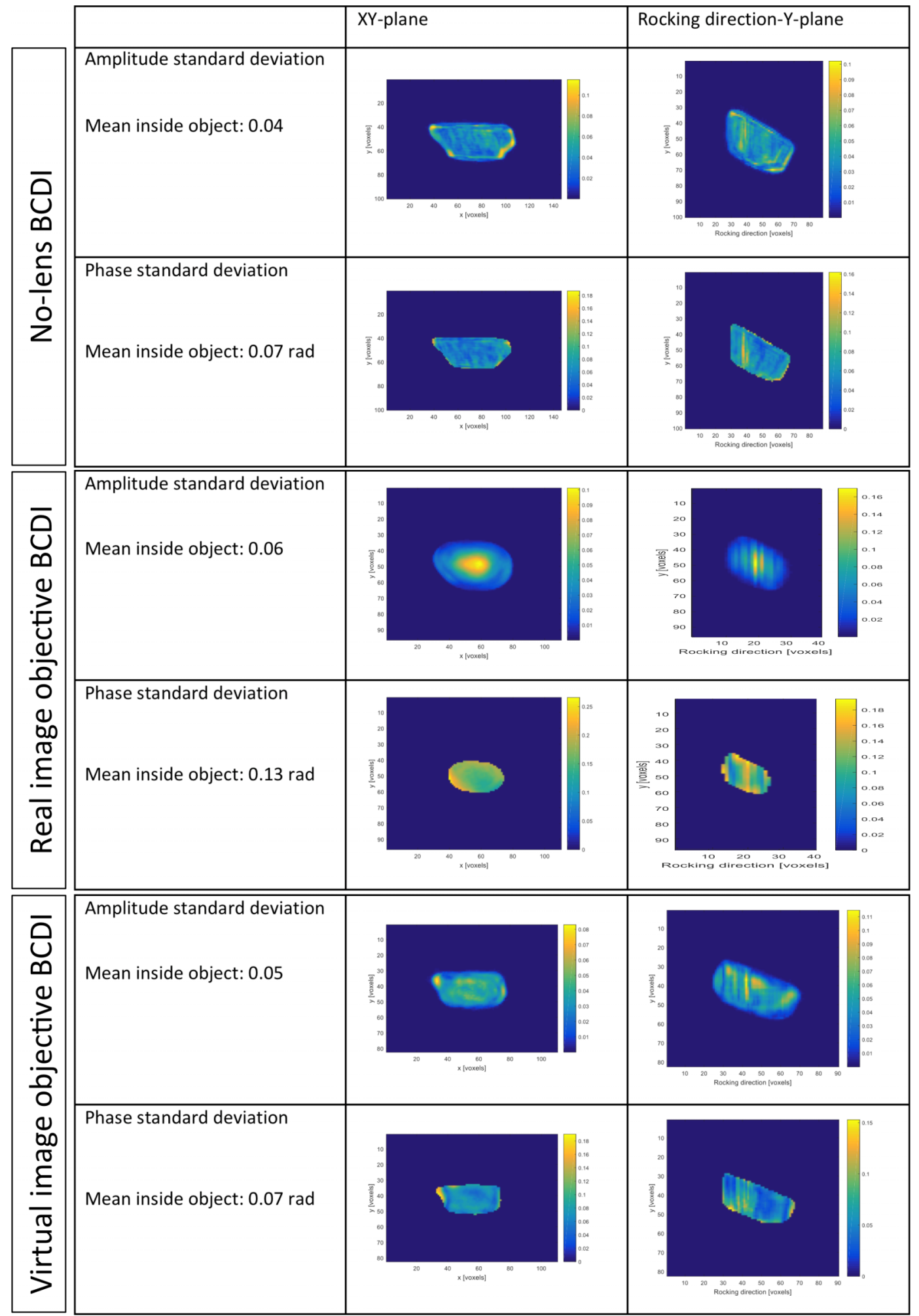

FIG. 8. Standard deviation observed among a set of BCDI reconstructions with different initial guesses. For each experiment [no-lens BCDI, objective BCDI (real and virtual plane)] and for two sections through the sample the standard deviations of the retrieved amplitude and phase are shown. 


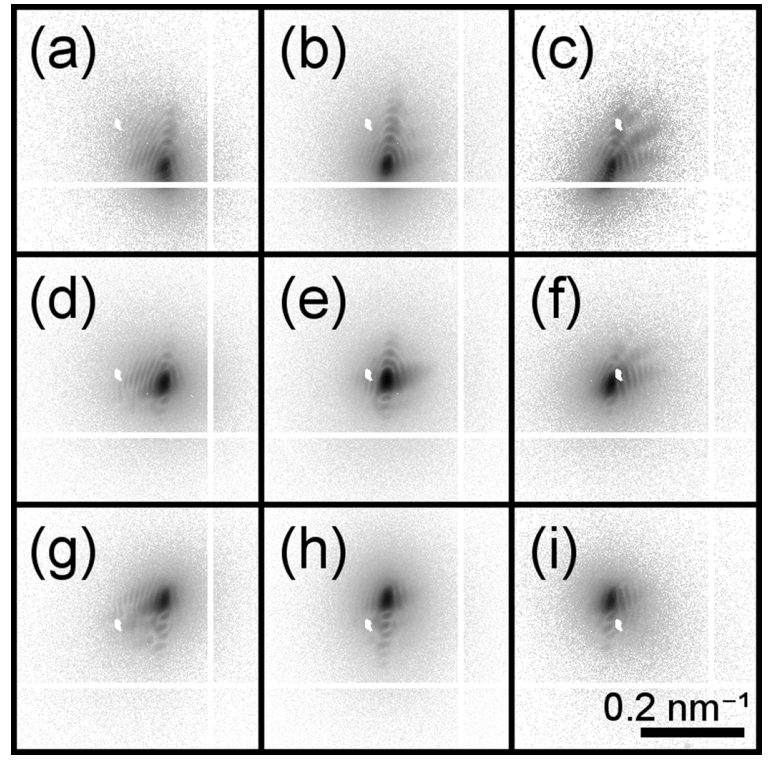

FIG. 9. The intensity pattern recorded at the central Bragg peak for the nine data sets used for Fourier synthesis. The images are plotted on a logarithmic scale covering six orders of magnitude. The rows correspond to steps in $2 \theta$ and the columns to steps in $\eta$ angle. The scale of all the images is the same with a common scale bar shown in (i).

taking the mean of the aligned reconstructions. As an initial seed for all $N=9$ independent reconstructions, we use the object that was recovered using only the central position of the lens. The image registration of the associated intensity patterns was determined by fitting the rocking curve and employing a mean square difference optimization. The shifts within the images were determined using the Fourier shift theorem and were therefore determined to a subpixel resolution. The intensity patterns were also normalized to match the Fourier amplitude between each data set. The ER algorithm was used to reconstruct the phase in the Fourier plane, and after each step a combined object was formed by averaging

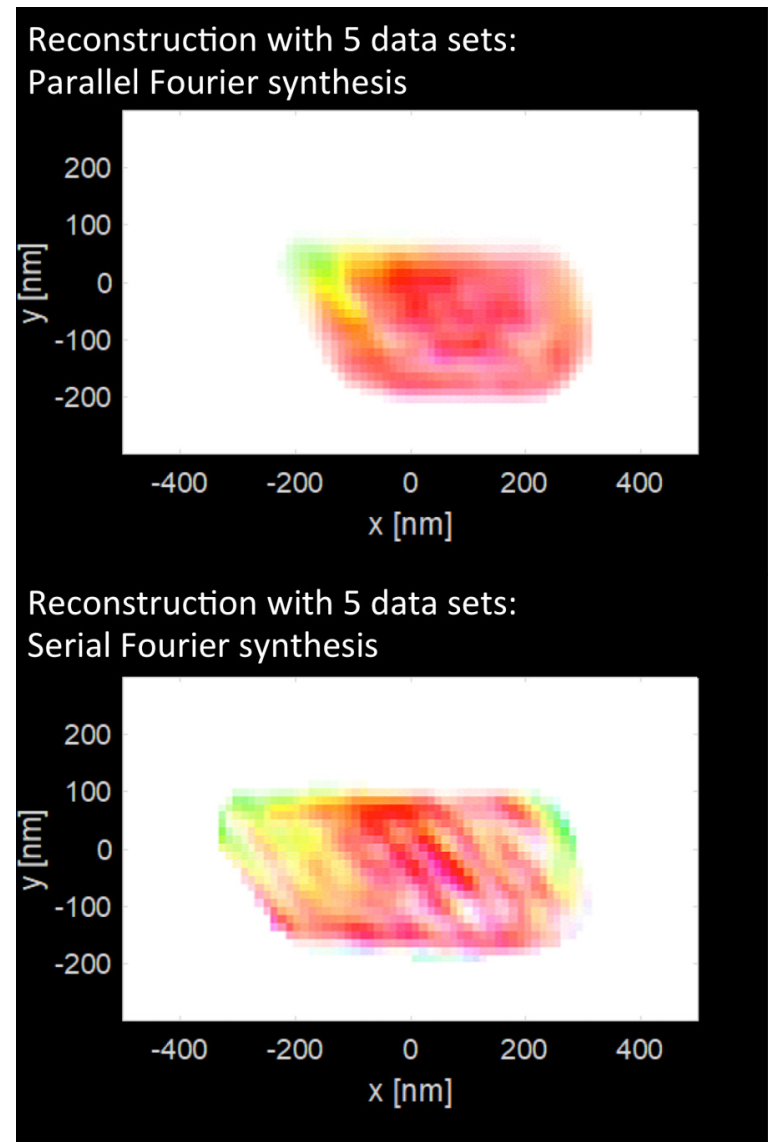

FIG. 10. Reconstruction results with small overlapping ratio. The top and bottom plots correspond to the object retrieved with only five data sets, corresponding to an overlapping ratio of about $40 \%-$ $50 \%$. The parallel and serial synthesis reconstructions are shown, respectively.

the nine retrieved objects. The shrinkwrap algorithm was used on this combined object. After 100 cycles the reconstruction had converged, and the Fourier reconstructions were averaged. The final object was found by inverting this synthesized Fourier amplitude and phase.

TABLE IV. Overlapping ratio among all pairs of Gaussian aperture positions (in percent). The overlapping ratio between two successive data sets is about $70 \%$ for the full data set and reduces to $45 \%$ when only the four outermost and one central data points are used.

\begin{tabular}{|c|c|c|c|c|c|c|c|c|c|}
\hline & Center & Top & Bottom & Left & Left top & Left bottom & Right & Right top & Right bottom \\
\hline Center & 100 & 58 & 65 & 72 & 38 & 49 & 75 & 39 & 51 \\
\hline Top & 58 & 100 & 14 & 46 & 72 & 11 & 48 & 74 & 12 \\
\hline Bottom & 65 & 14 & 100 & 43 & 9 & 73 & 44 & 9 & 74 \\
\hline Left & 72 & 46 & 43 & 100 & 59 & 62 & 30 & 17 & 18 \\
\hline Left top & 38 & 72 & 9 & 59 & 100 & 14 & 18 & 29 & 4 \\
\hline Left bottom & 49 & 11 & 73 & 62 & 14 & 100 & 18 & 4 & 29 \\
\hline Right & 75 & 48 & 44 & 30 & 18 & 18 & 100 & 59 & 61 \\
\hline Right top & 39 & 74 & 9 & 17 & 29 & 4 & 59 & 100 & 13 \\
\hline Right bottom & 51 & 12 & 74 & 18 & 4 & 29 & 61 & 13 & 100 \\
\hline
\end{tabular}




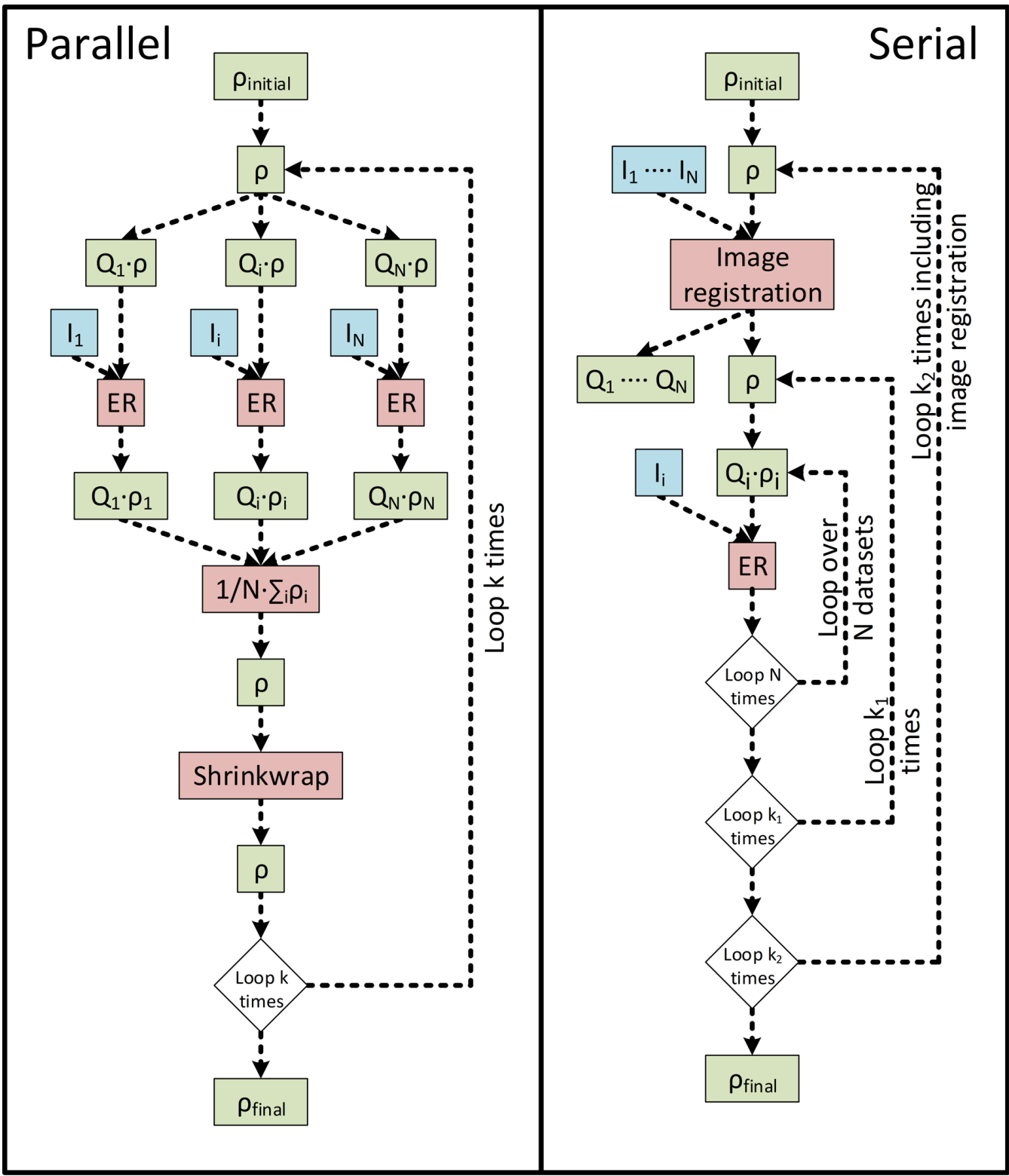

FIG. 11. Flow charts describing the numerical sequences involved in the Fourier synthesis reconstructions. The parallel and serial approaches are described. Green rectangles indicate operations on real-space variables, red rectangles are used for mathematical routines, and blue rectangles are used for the 3D experimental data. The $N$ overlapping data sets are identified by $I_{i}$ (with $1<i<\mathrm{N}$ ). The effective electron density is named $\rho$ and the phase ramps, which account, in real-space, for the shift associated to the nine overlapping data sets in reciprocal space are noted as $Q_{i}$. In our case $N=9, k=100$ (parallel), $k_{1}=20$ (serial), and $k_{2}=20$ (serial).

In the serial synthesis approach, sections of the global reconstructed Fourier plane are updated, accounting for the measurement information, in a serial fashion. Specifically, in an iterative scheme, it is based on updating the amplitude in Fourier space, weighted by the Gaussian pupil function, with the measured intensities for one data set at a time. The ER algorithm was used to enforce the support in between each updating cycle. Again, the initial seed was the reconstruction from the central data set. The iterative scheme is composed of three nested loops, one accounting for the $N$ individual data sets; another one producing the combined object; and an external one, referred to as image registration, for aligning the individual data sets.

\section{APPENDIX I: ROBUSTNESS OF THE FOURIER SYNTHESIS APPROACHES}

In order to confirm the robustness of our Fourier approaches, a series of numerical tests based on the experimental data have been performed. To this aim, the 3D real-space image obtained from the no-lens BCDI reconstruction was used as a test object. Its Fourier components were calculated, and the nine noise-free intensity data sets were extracted, applying the lens aperture truncation in all nine different locations of the reciprocal space. A first reconstruction was performed on the central diffraction pattern and further used to initialize the two Fourier synthesis reconstructions. 


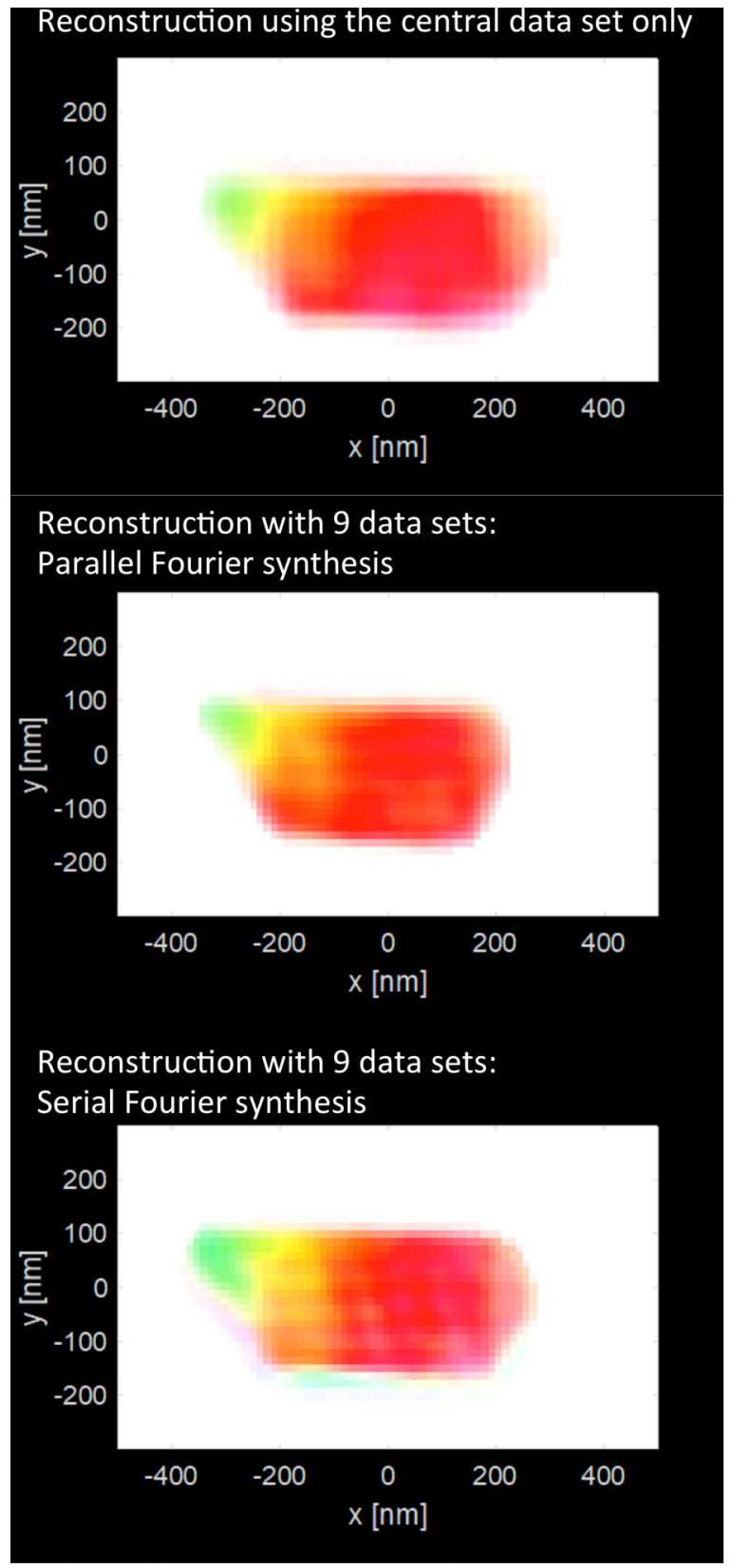

FIG. 12. Robustness of the Fourier synthesis approaches. Numerical tests performed on a data set arising from the real-space image obtained from the no-lens BCDI reconstruction, used to generate nine data sets, mimicking the nine shifted aperture measurements. The top reconstruction was obtained with classical BCDI on the central data set only while the middle and bottom reconstructions were obtained from Fourier synthesis using the nine data sets inverted with the parallel and serial approaches, respectively.

The corresponding results are shown in Fig. 12. The good agreement between the three reconstructions is a testament to

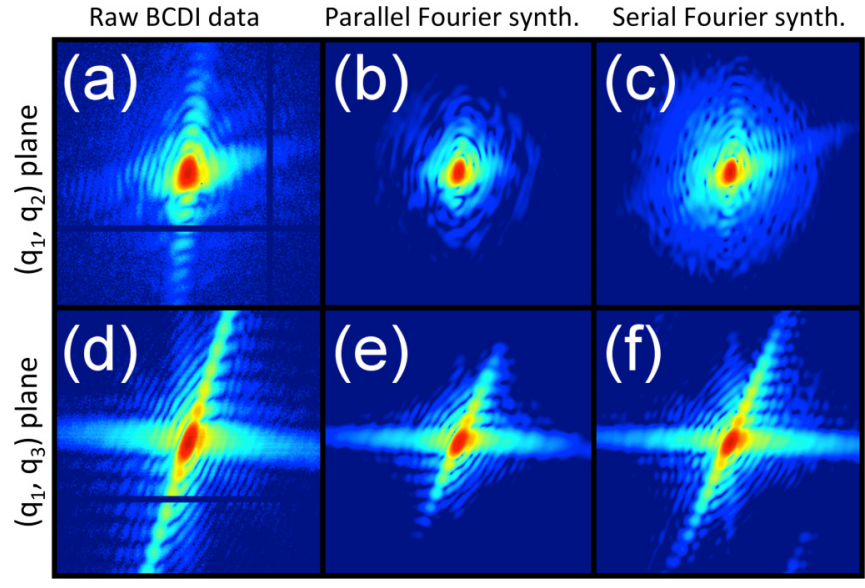

FIG. 13. Fourier synthesis reconstructions compared to BCDI raw data. (a), (d) Fourier space slices of the no-lens BCDI measured data compared to (b), (e) the parallel synthesis, and (c), (f) serial synthesis. (a)-(c) show the $\left(q_{1}, q_{2}\right)$ plane (i.e., same as Fig. 2) and (d)-(f) show the $\left(q_{1}, q_{3}\right)$ plane. The color scale is the same in all plots, $\log _{10}$ from 0 to 6 in intensity (one photon to 1000000 photons). The q-space range and scale is identical to Fig. 9.

the robustness of the Fourier synthesis approaches. There is a clear gain in spatial resolution observed when comparing the two synthesis approach reconstructions with the single data reconstruction.

\section{APPENDIX J: FOURIER SYNTHESIS: QUALITY OF THE RECONSTRUCTIONS IN RECIPROCAL SPACE}

This Appendix provides a detailed comparison between the intensity distributions retrieved by the two Fourier synthesis approaches with respect to the experimentally measured intensity. Figure 13 compares the retreived Fourier synthesis intensity and the intensity measured with the no-lens BCDI setup. It presents slices in the $\left(q_{1}, q_{2}\right)$ plane and in the $\left(q_{1}, q_{3}\right)$ plane, taken at the maximum of the Bragg peak. Figure 14 compares the Fourier synthesis reconstructions with the intensity measurements obtained in the central data set (no aperture shift). It presents the evolution of the $\left(q_{1}, q_{2}\right)$ intensity distribution as a function of the rocking curve scanning steps while Fig. 15 is a similar plot, taken in the $\left(q_{z}, q_{y}\right)$ plane as a function of the $q_{x}$ steps.

The figures reveal some differences in the behavior of these two algorithms. While the parallel Fourier synthesis approach reproduces pretty fairly the expected intensity distribution in the vicinity of the Bragg peak maximum, the agreement between the serial Fourier synthesis retrieved intensity and the BCDI measurement is less accurate in the same region. 


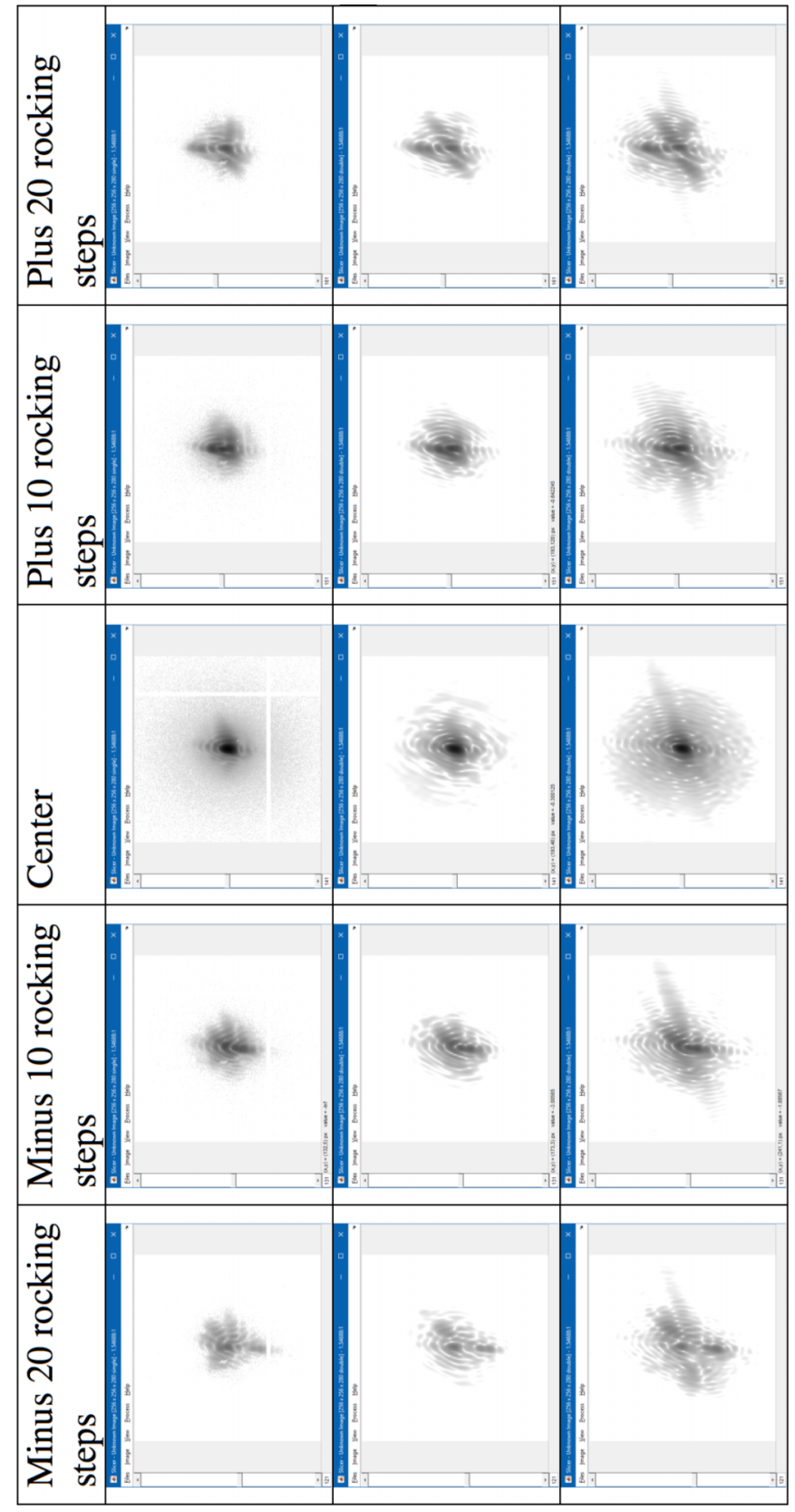

FIG. 14. Fourier synthesis reconstructions compared to the objective BCDI measurement (central data set). First column represents raw data, second column parallel synthesis, and third column serial synthesis. The intensity distribution in the $\left(q_{1}, q_{2}\right)$ plane is shown, as a function of steps on the rocking curve. The q-space range and scale is identical to Fig. 9.

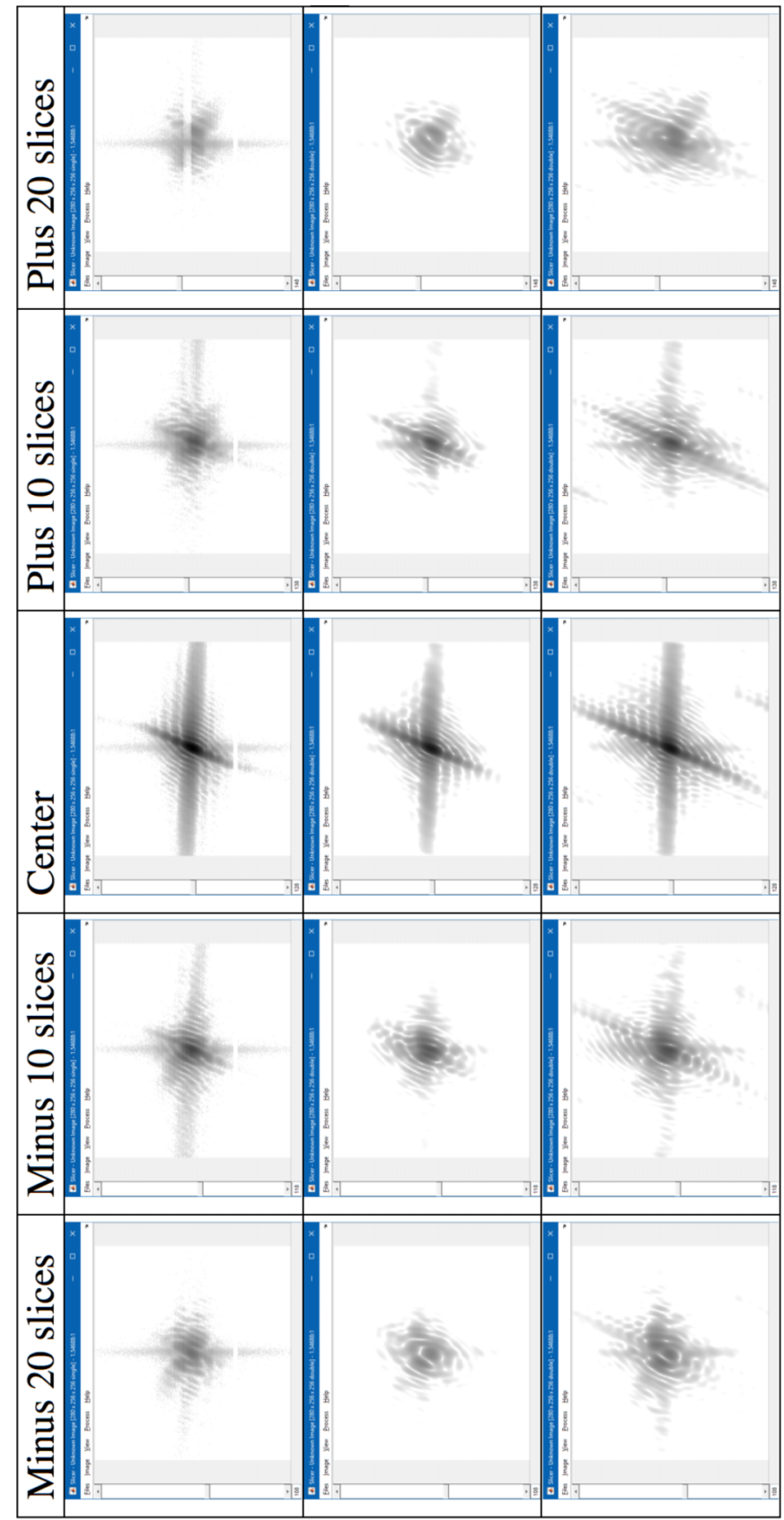

FIG. 15. Fourier synthesis reconstructions compared to the objective BCDI measurement (central data set). Complementary to results shown in Fig. 14, the plots represent the intensity distribution in the $\left(q_{z}, q_{y}\right)$ plane as a function of $q_{x}$. The q-space range and scale is identical to Fig. 9.
[1] D. B. Williams and C. B. Carter, Transmission Electron Microscopy (Plenum Press, New York, 1996).

[2] B. L. Adams, Ultramicroscopy 67, 11 (1997).

[3] H. F. Poulsen, J. Appl. Crystallogr. 45, 1084 (2012).

[4] W. Ludwig, S. Schmidt, E. M. Lauridsen, and H. F. Poulsen, J. Appl. Crystallogr. 41, 302 (2008).

[5] A. King, G. Johnson, D. Engelberg, W. Ludwig, and J. Marrow, Science 321, 382 (2008).
[6] U. Lienert, S. F. Li, C. M. Hefferan, J. Lind, R. M. Suter, J. V. Bernier, N. R. Barton, M. C. Brandes, M. C. Mills, M. P. Miller, B. Jakobsen, and W. Pantleon, JOM 63, 70 (2011).

[7] H. Simons, A. King, W. Ludwig, C. Detlefs, W. Pantleon, S. Schmidt, F. Stöhr, I. Snigireva, A. Snigirev, and H. F. Poulsen, Nat. Commun. 6, 6098 (2015).

[8] H. F. Poulsen, A. C. Jakobsen, H. Simons, S. R. Ahl, P. K. Cook, and C. Detlefs, J. Appl. Crystallogr. 50, 1441 (2017). 
[9] H. Simons, A. B. Haugen, A. C. Jakobsen, S. Schmidt, F. Stöhr, M. Majkut, C. Detlefs, J. E. Daniels, D. Damjanovic, and H. F. Poulsen, Nat. Mater. 17, 814 (2018).

[10] D. Sayre, Acta Crystallogr. 5, 843 (1952).

[11] J. Miao, P. Charalambous, J. Kirz, and D. Sayre, Nature 400, 342 (1999).

[12] H. Chapman and K. Nugent, Nat. Photonics 4, 833 (2010).

[13] J. Miao, T. Ishikawa, I. K. Robinson, and M. M. Murnane, Science 348, 530 (2015).

[14] M. A. Pfeifer, G. J. Williams, I. A. Vartanyants, R. Harder, and I. K. Robinson, Nature 442, 63 (2006).

[15] A. Yau, W. Cha, M. W. Kanan, G. B. Stephenson, and A. Ulvestad, Science 356, 739 (2017).

[16] V. Chamard, J. Stangl, G. Carbone, A. Diaz, G. Chen, C. Alfonso, C. Mocuta, and T. H. Metzger, Phys. Rev. Lett. 104, 165501 (2010).

[17] A. I. Pateras, M. Allain, P. Godard, L. Largeau, G. Patriarche, A. Talneau, K. Pantzas, M. Burghammer, A. A. Minkevich, and V. Chamard, Phys. Rev. B 92, 205305 (2015).

[18] F. Mastropietro, P. Godard, M. Burghammer, C. Chevallard, J. Daillant, J. Duboisset, M. Allain, P. Guenoun, J. Nouet, and V. Chamard, Nat. Mater. 16, 946 (2017).

[19] A. F. Pedersen, V. Chamard, and H. F. Poulsen, Opt. Express. 26, 23411 (2018).

[20] S. A. Alexandrov, T. R. Hillman, T. Gutzler, and D. D. Sampson, Phys. Rev. Lett. 97, 168102 (2006).

[21] A. Snigirev, V. Kohn, I. Snigireva, and B. Lengeler, Nature 384, 49 (1996).
[22] J. R. Fienup, Appl. Opt. 21, 2758 (1982).

[23] W. Cha, S. Song, N. C. Jeong, R. Harder, K. B. Yoon, I. K. Robinson, and H. Kim, New J. Phys. 12, 035022 (2010).

[24] S. Marchesini, H. He, H. N. Chapman, S. P. Hau-Riege, A. Noy, M. R. Howells, U. Weierstall, and J. C. H. Spence, Phys. Rev. B 68, 140101(R) (2003).

[25] G. Zheng, R. Horstmeyer, and C. Yang, Nat. Photonics 7, 739 (2013).

[26] C. Detlefs, M. A. Beltran, J.-P. Guigay, and H. Simons, J. Synchrotron Rad. 27, 119 (2020).

[27] K. Wakonig, A. Diaz, A. Bonnin, M. Stampanoni, A. Bergamaschi, J. Ihli, M. Guizar-Sicairos, and A. Menzel, Sci. Adv. 5, eaav0282 (2019).

[28] C. Liu, S. Marchesini, and M. K. Kim, Opt. Express 22, 17830 (2014).

[29] A. J. Morgan, M. Prasciolu, A. Andrejczuk, J. Krzywinski, A. Meents, D. Pennicard, H. Graafsma, A. Barty, R. J. Bean, M. Barthelmess, D. Oberthuer, O. Yefanov, A. Aquila, H. N. Chapman, and S. Bajt, Sci. Rep. 5, 9892 (2015).

[30] K. T. Murray, A. F. Pedersen, I. Mohacsi, C. Detlefs, A. J. Morgan, M. Prasciolu, C. Yildirim, H. Simons, A. C. Jakobsen, H. N. Chapman, H. F. Poulsen, and S. Bajt, Opt. Express 27, 7120 (2019).

[31] D. Shapiro, P. Thibault, T. Beetz, V. Elser, M. Howells, C. Jacobsen, J. Kirz, E. Lima, H. Miao, A. M. Neiman, and D. Sayre, Proc. Natl. Acad. Sci. USA 102, 15343 (2005).

[32] M. J. Cherukara, W. Cha, and R. J. Harder, Appl. Phys. Lett. 113, 203101 (2018). 